\title{
RETRACTED ARTICLE: Residual effect of two chemical insecticides on functional response of Habrobracon hebetor say (Hymenoptera: Braconidae) to Ephestia kuehniella Zeller (Lepidoptera: Pyralidae)
}

\author{
Azadeh Jarrahi ${ }^{1} \cdot$ Seyed Ali Safavi ${ }^{1}$
}

Received: 5 April 2020 / Accepted: 31 August 2020 / Published online: 2 September 2020

(C) African Association of Insect Scientists 2020

The authors have retracted this article [1] because it significantly overlaps with a previously published article [2] by the same authors without proper citation. Both authors agree with this retraction. The online version of this article contains the full text of the retracted article as Supplementary Information.

\section{References}

1. Jarrahi, A., Safavi, S.A. Residual effect of two chemical insecticides on functional response of Habrobracon hebetor say (Hymenoptera: Braconidae) to Ephestia kuehniella Zeller (Lepidoptera: Pyralidae). Int J Trop Insect Sci (2020). https://doi.org/10.1007/s42690-02000245-1

2. Jarrahi, A., Safavi, S.A. Parasitism Rate of Habrobracon hebetor to Ephestia kuehniella Larvae: Residual Effect of Deltamethrin, Fenvalerate and Azadirachtin. J Insect Behav 29, 548-556 (2016). https://doi.org/10.1007/s10905-016-9583-z

Supplementary Information The online version contains supplementary material available at https://doi.org/10.1007/s42690-02000245-1.

Seyed Ali Safavi

a.safavi@urmia.ac.ir

1 Department of Plant Protection, Faculty of Agriculture, Urmia University, Urmia, Iran 